\title{
VIII. An account of some experiments and observations on the constituent parts of certain astringent vegetables; and on their operation in tanning
}

\author{
Humphry Davy Esq.
}

To cite this article: Humphry Davy Esq. (1803) VIII. An account of some experiments and observations on the constituent parts of certain astringent vegetables; and on their operation in tanning, Philosophical Magazine Series 1, 17:65, 63-76, DOI: $10.1080 / 14786440308676372$

To link to this article: http://dx.doi.org/10.1080/14786440308676372

$$
\text { 曲 Published online: } 18 \text { May } 2009 .
$$

Submit your article to this journal $\sqsubset$

$$
\text { Џlll Article views: } 3
$$




\section{$\left[\begin{array}{lll}83 & ]\end{array}\right.$}

VIII. An Account of some Experiments and Observations on the constituent Parts of certain astringent Vegetables; and on their Operation in Tanning. By HUMPHRY Davy, Esq., Professor of Chemistry in the Royal Institution.** $\mathrm{T}_{\mathrm{HE}}$ discovery made by $\mathrm{M}$. Seguin, of a peculiar vegetable matter which is essential to the tanning of skin, and which is possessed of the property of precipitating gelatine from its solutions, has added considerably to our knowledge of the constituent parts of astringent vegetables.

Mr. Proust has investigated many of the properties of this substance; but though his labours, and those of other chemists, have led to various interesting observations, yet they are far from having exhausted the subject. The affinities of tannin have been hitherto very little examined; and the manner in which its action upon animal matters is modified by combination with other substances, has been scarcely at all studied.

At the desire of the managers of the Royal Institution, I began, in September 1801, a series of experiments on the substances employed in the process of tanning, and on the chemical agencies concerned in it. These experiments have occupied, ever since, a considerable portion of my leisure hours; and I now presume to lay before the Royal Society an account of their general results. My chief design was, to attempt to elucidate the practical part of the art : but in pursuing it, I was necessarily led to general chemical inquiries, concerning the analysis of the different vegetable substance -ontaining tannin, and their peculiar properties.

1. Observations on the Analysis of astringent Vegetable Infusions.

The substances that have been supposed to exist most gencrally in astringent infusions are, tannin, gallic acid, and extractive matter.

The presence of tannin in an infusion, is denoted by the precipitate it forms with the solution of glue or of isinglass. And, when this principle is wholly separated, if the remaining liquor gives a dark colour with the oxygenated salts of iron, and an immediate precipitate with the solutions of alum and of muriate of tin, it is believed to contain gallic acid and extractive matter.

The expcriments of MM. Fourcroy, Vauquelin, and Se-

is From Philosnphical Transactions for 1803 .

guin, 
guin, have shown that many astringent solutions undergo a change by exposure to the atmosphere; an insoluble matter being precipitated from them. A precipitation is likewise occasioned in them by the action of heat; and these circumstances render it extremely difficult to ascertain, with any degree of precision, the quantities of their constituent parts, as they exist in the primitive combination.

After trying several experiments on different methods of ascertaining the quantity of tannin in astringent infusions, I was induced to employ the common process of precipitation by gelatine, as being the most accurate.

This process, however, requires many precautions. The tanning principle in different vegetables, as will be seen hereafter, demands for its saturation different proportions of gelatine; and the quantity of the precipitate obtained by filtration is not always exactly proportional to the quantities of tannin and gelatine in solutions, but is influenced by the degree of their concentration. Thus $I$ found that 10 grains of dry isinglass, dissolved in two ounces of distilled water, gave, with solution of galls in excess, a precipitate weighing, when dry, 17 grains; whilst the same quantity, dissolved in six ounces of water, produced, all other circumstances being similar, not quite 15 grains. With more diluted solutions, the loss was still greater; and analogous effects took place, when equal portions of the same solution of isinglass were acted on by equal portions of the same infusion of galls diluted in different degrees with water; the least quantity of precipitate being always produced by the least concentrated liquor. In all cases, when the weak solutions were used, it was observed that the residual fluid, though passed two or three times through the filter, still remained more or less turbid and opaque; so that it is most likely that the deficiency arose from the continued suspension of some of the minutely divided solid matter in the liquid mass.

The solutions of gelatine, for the purposes of analysis, should be employed only when quite fresh, and in as high a state of saturation as is compatible with their perfect fluidity. 1 have observed that, in cases when they approach towards the state of jelly, their power of acting upon tannin is materially altered, and they produce only a very slight precipitation. As the degree of fluidity of solutions of gelatine is influenced by their temperature, I have found it expedient, in all comparative experiments, to bring them, and the astringent infusions on which they are designed to act, as nearly as possible to a common degree of heat. My standard 
standard temperature has been between $60^{\circ}$ and $70^{\circ}$ Fuhrenheit; and the solutions of gelatine that I have used were made by dissolving 120 grains of isinglass in 20 ounces of water.

In ascertaining the proportions of tannin in astringent infusions, great care must be taken to prevent the presence of any excess of gelatine; for when this excess 'exists, I have found that a small portion of the solid compound formed is redissolved, and the results of the experiment otherwise affected. It is not difficult to discover the precise point of saturation, if the solution of isinglass be added only in small quantities at a tume, and if portions of the clear liquor be passed through a filter at different periods of the process. The properties of these portions will indicate the quantities of the solution of gelatine required for the completion of the expcriment.

That the composition of any precipitate containing tannin and gelatine may be known with a tolerable degree of precision, it is necessary that the isinglass cmployed in the solution, and the new compound formed, be brought as nearly as possible to the same degree of dryness. For this purpose I have generally exposed them, for an equal time, upon the lower plate of a sand-bath, which was seldom licated to more than $150^{\circ}$. This method I have found much better than that of drying at the temperatures of the atmosphere, as the different states of the air, with regard to moisture, materially influence the results.

Mr. Hatchett has noticed, in his excellent paper on Znophytes, \&e.**;, that isinglass is almost wholly composed of grelatine. I have found that 100 grains of good and dry isinglass contain rather morc than 98 grans of matter solible in water. So that when the quantity of isinglass, in any solution employed for acting upon an astringent infusion, is compared with the quantity of the precipitate obtained, the difference between them will indicate the proportion of tannin, as it exists in the combination.

After the tannin has been scparated from an astringent infusion, for the purpose of ascercainmy its otier component parts, I have becn accustomed to evaporate the residual liquor very slowly, at a temperature below $200^{\circ}+$. In

* Philosophical Transactions for 1800, P. 327.

+ M. Deyeux his shown (Annales de. C-2mb tome xrit. p. 36), that in

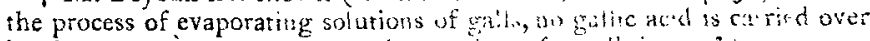

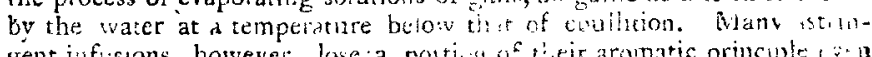
gent iafustons, however, lose a pontion if the ter arwatic princuple $: a$ in cases when thev are not made to bol ; but this substance, thong! .....

FoL. XVII. No. 65 . 
this process, if it contains extractive matter, that substance is in part rendered insoluble, so as to fall to the bottom of the vessel. When the fluid is reduced to a thick consistence, I pour alcohol upon it. If any gallic acid or soluble extractive matter be present, they will be dissolved, after a little agitation, in the alcohol; whilst the mucilage, if any exist, will remain unaltered, and may be separated from the insoluble extract by lixiviation with water.

I have made many experiments with the hope of discovering a method by which the respective quantities of gallic acid and extractive matter, when they exist in solution in the alcohol, may be ascertained, but without obtaining success in the results. It is impossible to render the whole of any quantity of extractive matter insoluble by exposure to heat and air, without at the same time decomposing a portion of the gallic acid. That acid cannot be sublimed without being in part destroyed; and, at the temperature of its sublimation, extractive matter is wholly converted into new products.

Ether dissolves gallic acid ; but it has comparatively little action upon extractive matter. I have been able, in examining solutions of galls, to separate a portion of gallic acid by means of ether. But when the extractive matter is in large quantities, this method does not succeed, as, in consequence of that affinity which is connected with mass*, the greatest part of the acid continues to adhere to the extract.

Alumine has a strong attraction for extractive matter, but comparatively a weak one for gallic acid + . When carbonate of alumine is boiled for some time with a solution containing extractive matter, the extractive matter is wholly taken up by the earth, with which it forms an insoluble compound; but into this compound some of the gallic acid appears likewise to enter; and the portion remaining dissolved in the solution is always combined with alumine.

I have not, in any instance, been able to separate gallic acid and extractive matter perfectly from each other ; but I have generally endeavoured to form some judgment concerning their relative proportions, by means of the action of the salts of alumine and the oxygenated salts of iron. Muriate of alumine precipitates much of the extractive

dent to the smell, in the water that comes orer, cannot be detected by chemical reagents.

* See Berthollet, Rechercbes sw les Lois de l'Affinití. Mem. de l'lnstitute National, tome iii. p. :.

+ See Fiedler, Fournal de Chimie, par J. B. Van Mons, tome i. p. Se. 
matter from solutions, without acting materially upon gallic acid; and, after this precipitation, some idea may be formed concerning the quantity of the gallic acid by the colour it gives with the oxygenated sulphate of iron. In this process, however, great care must be taken not to add the solution of the sulphate of iron in excess; for in this case the black precipitate formed with the gallic acid will be redissolved, and a clear olive-coloured fluid only will be obtained.

The saline matters in astringent infusions adhere so strongly to the vegetable principles, that it is impossible to ascertain their nature, with any degree of accuracy, by means of common reagents. By incineration of the products obtained from the evaporation of astringent infusions, I have usually procured carbonate of lime and carbonate of potash.

In the different analyses, as will be secn from the results given in the following sections, I have attended chiefly to the proportions of the tanning principle, and of the principles precipitable by the salts of iron, as being most connected with practical applications.

With regard to the knowledge of the nature of the different substances, as they exist in the primitive astringent infusion, we can gain, by our artificial methods of examination, only very imperfect approximations. In acting upon them by reagents we probably, in many cases, alter their nature; and very few of them only can be obtained in an uncombined state. The comparison, however, of the products of different experiments with each other is always connected with some useful conclusions; and the accumulation of facts with regard to the subject must finally tend to elucidate this obscure but most interesting part of chemistry.

\section{Experiments on the Infusions of Galls.}

$I$ have been very much assisted, in my inquiries concerning the properties of the infusions of galls, by the able memoir of M. Deyeux on Galls*.

The strongest infusion of galls that I could obtain at $56^{\circ}$ Fahrenheit, by repeatedly pouring distilled water upon the best Aleppo galls broken into small pieces, and suffering it to remain in contact with them till the saturation was complete, was of the specific gravity 1.068. Four hundred grains of it produced, by evaporation at a temperature be-

* Annales de Chimie, ome xvii. p. I. E. 2 
low $200^{\circ}$, fifty-three grains of solid matter; which, as well as I could estimate by the metbods of analysis that have been just described, consisted of about nine-tenths of tannin, or matter precipitable by gelatine, and one-tenth of gallic acid, united to a minute portion of extractive matter.

100 grains of the solid matter obtained from the infusion left, after incineration, nearly $4 \frac{3}{4}$ grains of ashes; which were chiefly calcareous matter, mixed with a small portion of fixed alkali. The infusion strongly reddened paper tinged with litmus. It was semitransparent, and of a yellowishbrown colour. Its taste was highly astringent.

When sulphuric acid was poured into the infusion a dense whitish precipitate was produced; and this effect was constant, whatever quantity of the acid was used. The residual liquor, when passed through the filter, was found of a shade of colour deeper than before. It precipitated gelatine, and gave a dark colour with the oxygenated sulphate of iron.

The solid matter remaining on the filter slightly reddened vegetable blues; and, when dissolved in warm water, copiously precipitated the solutions of isinglass. M. Proust who first paid attention to its properties, supposes that it is a compound of the acid with tannin : bat I suspect that it also contains gallic acid, and probably a small portion of estractive matter. This last substance, as is well known, is thrown down from its solutions by sulphuric acid; and I found, in distilling the precipitate from galls by sulphuric acid, at a heat abore $212^{\circ}$, that a fluid came over of a lightyellow colour, which was rendered black by oxygenated sulphate of iron, but which was not altered by gelatine.

Muriatic acid produced, ir: the infusion, effects analogous to those produced by sulphuric acid; and two compotinds of the acid and the vegetable substances were formed : the one united to excess of acid, which remained in solution; the other containing a considerable quantity of tamin, which was precipitated in the solid form.

When concentrated nitric acid was made to act upon the infusion, it was rendered turbid; but the solid matter formed was immediately dissolved with effervescence, and the liquor then became clear, and of an orange colour. On examining it, it was found that both the tannin and the gallic acid wcre destroyed; for it gave no precipitate either with gelatine or the salts of iron, even after the residual nitric acid was saturated by an alkali. By evaporation of a portion of

* The fact of the precipitation of the solution of galls by acids was not cud by M. Dizé. See Annates de Cbimie, tome xxxy. p. 37 . 
the fluid a soft substance was obtained, of a yellowishbrown colour, and of a slightly sourish taste. It was soluble in water, and precipitated the nitro-muriate of tin, and the nitrate of alumine; so that its properties approached to those of extractive matter; and it probably contained oxalic acid, as it rendered turbid a solution of muriate of lime.

When a very weak solution of nitric acid was mixed with the infusion, a permanent precipitate was formed; and the residual liquor, examined by the solution of gelatine, was found to contain tannin.

A solution of pure potash was poured into a portion of the infusion. At first a faint turbid appearance was perceived; but by agitation the fluid became clear, and its colour changed from yellow-brown to brown-red; and this last tint was most vivid on the surface, where the solution was exposed to the atmosphere. The solution of isinglass did not act upon the infusion modified by the alkali till an acid was added in excess, when a copious precipitation was occasioned.

The compound of potash and solution of galls, when evaporated, appeared in the form of an olive-coloured mass, which had a faint alkaline taste, and which slowly deliquesced when exposed to the air.

Soda acted upon the infusion in the same manner as potash; and a fluid was formed of a red-brown colour, which gave no precipitate to gelatine.

Solution of ammonia produced the same colour as potash and soda, and formed so perfect an union with the tannin of the infusion that it was not acted upon by gelatine. When the compound liquor was exposed to the beat of boiling water, a part of the ammonia flew off, and another part reacted upon the infusion so as to effect a material change in its properties. A considerable quantity of insoJuble matter was formed, and the remaining liquor contained little tamin and gallic acid, but a considerable portion of a substance that precipitated muriate of tin and the salts of alumine.

When the experiment on the ebullition of the compound of the infusion and ammonia was made in close vessels, the liquor that came over was strongly impregnated with ammonia; its colour was light vellow, and, when saturated with an acid, it was very little altered by the salts of iron. The residual fluid after the process had been continued for some time, as in the other case, precipitated gelatine slightly, but the salts of alumine copiously; and it gave a tinge of red to litmus paper. 
When solution of lime, of strontia, or of barytes, was poured in excess into a portion of the infusion, a copious olive-coloured precipitate was formed, and the solution became almost clear, and of a reddish tint. In this case the tannin, the gallic acid, and the extractive matter, seemed to be almost wholly carried down in the precipitates; as the residual fluids, when saturated by an acid, gave no precipitate to gelatine, and only a very slight tint of purple to oxygenated sulphate of iron.

When the solutions of the alkaline earths were used only in small quantities, the infusion being in excess, a smaller quantity of precipitate was formed, and the residual liquor was of an olive-green colour; the tint being darkest in the experiment with the barytes, and lightest in that with the lime. This fluid, when examined, was found to hold in solution a compound of gallic acid and alkaline earth. It became turbid when acted on by a little sulphuric acid; and, after being filtrated, gave a black colour with the solutions of iron, but was not acted upon by gelatine.

When a large proportion of lime was heated for some time with the infusion, it combined with all its constituent principles, and gave, by washing, a fluid which had the taste of lime water, and which held in solution only a very small quantity of vegetable matter. Its colour was pale yellow; and, when saturated with muriatic acid, it did not precipitate gelatine, and gave only a slight purple tinge to the solutions of the salts of iron. "The lime in combination with the solid matter of the infusion was of a fawn colour. It became grcen at its surface, where it was exposed to the air; and, when washed with large quanties of water, it continued to give, even to the last portions, a pale yellow tinge.

Magnesia was boiled in one portion of the infusion for a few hours; and mixed in excess with another portion, which was suffered to romain cold. In both cases a deep green fluid was obtained, which precipitated the salts of iron but not the solutions of gelatine; and the magnesia had acquired a grayish-green tint. Watcr poured upon it became green, and accuired the propertics of the fluid at first obtained. After long washing the colour of the magnesia changed to dirty yellow; and the last portions of water made to act upon it were pale yollow, and altered very little the solutions of iron.

When the magnesia was dissolved in muriatic acid, a brownish and turbid flaid was obtained, which precipitated gelatine and the oxygenated. salts of iron. So that there is every reason to beliese that the earth, in acting on the astringent 
astringent infusion, had formed two combinations; one containing chiefly gallic acid, which was easily soluble in water; the other containing chiefly tannin, which was very difficultly soluble.

Alumine boiled with the infusion became yellowish-gray, and gave a clear white fluid, which produced only a tinge of light purple in the solutions of iron. When the earth* was employed in a very small quantity, however, it formed an insoluble compound only with the tannin and the extract, and the residual liquor was found to contain a gallate of alumine with excess of acid.

The oxides of tin and of zinc, obtained by nitric acid, were boiled with separate portions of the infusion for two hours. In both cases a clear fluid, which appeared to be pure water, was obtained; and the oxides gained a tint of dull yellow. A part of each of them was dissolved in muriatic acid. The solution obtained was yellow; it copiously precipitated gəlatine, and gave a dense black with the salts of iron. Mr. Proust $t$, who first observed the action of oxide of tin upon astringent infusions, supposes that portions of tannin and gallic acid are decomposed in the process, or converted, by the oxygen of the oxide, into new substances. These experiments do not, however, appear to confirm the supposition.

M. Deyeux observed that a copious precipitation was occasioned in infusion of galls, by solutions of the alkalis combined with carbonic acid. Mr. Proust has supposed that the solid matter formed is pure tannin, separated from its solution by the stronger affinity of the alkali for water; and he recommends the process as a method of obtaining tannin.

In examining the precipitate obtained by carbonate of potash fully combined with carbonic acid, and used to saturation, I have not been able to recognise in it the properties which are usually ascribed to tannin : it is not possessed of the astringent taste, and it is but slightly soluble in cold water or in alcohol. Its solution acts very little upon gelatine, till it is saturated with an acid; and it is not possessed of the property of tanning skin.

In various cases in which the greatest care was taken to use no excess, either of the astringent infision or of the alkaline solution, I have found the solid matter obtained possessed of analogous properties; and it has always given,

* Mr. Fiedler, I believe, first observed the action of alumine upon tannin.-Van Mou's Journal, vol. i. p. 86.

$\uparrow$ Annales de Cbimie, tome xlii. p. 69. 
by incincration, a considerable portion of carbonate of potash, and a small quantity of carbonate of lime.

'i he fluid remaining after the separation of the precipitate was of a dark brown colour, and became green at the surface when it was exposed to the air. It gave no precipitate to solution of gelatine, and afforded only an olivecoloured precipitate with the salts of iron.

When murlatic acid was poured into the clear fluid, a violent effervescence was produced; the fluid became turbid; a precipitate was deposited; and the residual liquor actid upon gelatine and the salts of iron in a manner similar to the primitive infusion.

M. Deyeux, in distilling the precipitate from infusion of galls by carbonate of potash, obtained crystals of gailic acid. In following his process, I had similar results; and a fluid came over which reddened litmus paper, and precipitated the salts of iron black, but did not act upan gelatine.

When the precipitate by carbonate of potash was acted upon by warm water, applied in large quantities, a considerable pertion of it was dissolved; but a part remained which could not in any way be made to enter into solution, and its properties were very different from those of the entire precipitate. It was not at all affected by alcohol : it was acted on by muriatic acid, and partially dissolved; and the solution precipitated gelatine and the salts of iron. It afforded, by incineration, a considerable portion of lime, but no alkali.

In comparing these facts it would scem that the precipitate from infusion of galls consists partly of tannin and gallic acid united to a small quantity of alkali, and partly of these vegetable matters combined with calcareous earth; and it will appear probable, when the facts hereafter detailed are examined, that both the potash and the lime are con. tained in these compounds in a state of union with carbonic acid.

The solutions of carbonate of soda and of carbonate of ammonia, both precipitated the infusion of galls in a manner similar to the carbonate of potash; and each of the precipitates, when acted on by boiling water, left a small quantity of insoluble matter, which seemed to consist chiefly of tannin and carbonate of lime.

The entire precipitare by carbonate of soda produced, wh in incincrated, carbonate of soda and carbonate of lime. The precipitate by carbonate of ammonia, when exposed to 2. heat sufficient to boil water, in a returt having a receiver 
attached to it, gave out carbonate of ammonia, (which was condensed in small crystals in the neck of the retort,) and a yellowish fluid, which had the istrong smell and taste of this volatile salt. After the process of distillation, the solid matter remaining was found of a dark brown colour; a part of it readily dissolved in cold water, and the solution acted on gelatine.

The residual fluid of the portions of the infusion which had been actcd on by the carbonates of soda and of ammonia, as in the instance of the carbonate of potash, gave no precipitate with gelatine, till they were saturated with an acid; so that in all these cases the changes are strictly analogrous.

The infusion of galls, as appears from the analysis, contains in its primitive state calcareous matter. By the action of the mild alkalis, this substance is preciptated in union with a portion of the vegetable matter, in the form of an insoluble compound. The alkalis themselves at the same time enter into actual combination with the remaining tannin and gallic acid; and a part of the compound formed is precipitated, whilst another part remains in solution.

When the artificial carbonates of lime, magnesia, and barytes, were separately boiled with the portions of infusion of galls for some hours, they combined with the tannin contained in it so as to form with it insoluble compounds; and in each case a deep green fluid was obtained, which gave no precipitate to gelatine even when an acid avas added, but which produced a decp black colour in the solutions of the salts of iron.

Sulphate of lime, when finely divided, whether natural or artificial, after having been long heated with a small quantity of the infusion, was found to have combined with the tannin of it, and to have ganced a faint tinge of light brown. The liquid became of a blue-green colour, and acted upon the salts of iron, but not upon gelatine; and there is every reason to suppose that it held in solution a triple compund, of gallic acid, sulphuric acid, and lime.

We owe to Mr. Proust the discovery that different solutions of the neutral salts precipitate the infusion of galls; and he supposes that the precipiation is owing to their combining with a portion of the water which held the vegetable matter in solution. In examining the solid matters thrown down from the infusion by sulphate of alumine, nitrate of potash, acctite of potash, muriate of soda, and muriate of barytes, I found thein soluble, to a certain extent, 
tent, in water, and possessed of the power of acting upon gelatine. From the products given by their incineration and by their distillation, I am, however, inclined to believe that they contain, besides tannin, a portion of gallic acid and extractive matter, and a quantity of the salt employed in the primitive solution.

It is well known that many of the metallic solutions occasion dense precipitates in the infusion of galls; and it has been generally supposed that these precipitates are composed of tannin and extractive matter, or of those two substances and gallic acid united to the metallic oxide; but from the observation of different processes of this kind, in which the salts of iron and of tin were employed, I am inclined to believe that they contain also a portion of the acid of the saline compound.

When the muriate of tin was made to act upon a portion of the infusion, till no more precipitation could be produced in it, the fluid that passed through the filter still acted upon gelatine, and seemed to contain no excess of acid; for it gave a precipitate to carbonate of potash without producing effervescence. The solid compound, when decomposed by sulphuretted hydrogen, after the manner recommended by Mr. Proust, was found strongly to redden litmus paper, and it copiously precipitated nitrate of silver; whereas the primitive infusion only rendered it slightly turbid; so that there is every reason to believe that the precipitate contained muriatic acid.

By passing the black and turbid fluid, procured by the action of solution of oxygenated sulphate of iron in excess upon a portion of the infusion, through finely-divided pure flint, contained in four folds of filtrating paper, I obtained a light olive-green fluid, in which there was no excess of sulphuric acid, and which I am inclined to suppose was a solution of the compound of gallic acid and sulphate of iron, with superabundance of metallic salt. I have already mentioned that gallic acid, when in very small proportion, does not precipitate the oxygenated salts of iron; and Mr. Proust, in his ingenious paper upon the Difference of the Salts of Iron, has supposcd that, in the formation of ink, a portion of the oxide of iron in union with gallic acid is dissolved by the sulphuric acid of the sulphate. This comes near to the opinion that they form a triple compound; and, in reasoning upon the gencral phænomena, it seems fair to conclude that, in the case of the precipitation of tamin by the salts of tin and of iron, compounds are formed of tannin

and 
and the salts ; and that, of these compounds, such as contain tin are slightly soluble in water, whilst those that contain iron are almost wholly insoluble.

In examining the action of animal substances upon the infusion of galls, with the view of ascertaining the composition of the compounds of gelatine and of skin with tansin, I found that a saturated solution of gelatine, which contained the soluble matter of 50 grains of dry isinglass, produced from the infusion a precipitate that weighed nearly 91 grains; and in another instance, a solution containing 30 grains of isinglass gave about 56 grains; so that, taking the mean of the two experiments, and allowing for the small quantity of insoluble matter in isinglass, we may conclude that 100 grains of the compound gelatine and tannin, formed by precipitation from saturated solutions, contain about 54 grains of gelatine, and 46 of tannin.

A piece of dry calf-skin, perfectly free from extraneous matter, that weighed 180 grains, after being prepared for tanning by long immersion in water, was tanned in a portion of the infusion, being exposed to it for three weeks. When dry, the leather weighed 295 grains; so that, considering this experiment as accurate, leather quickly tanned by means of an infusion of galls consists of about 61 grains of skin, and 39 of vegetable matter, in 100 grains.

After depriving a portion of the infusion of all its tanning matter, by repeatedly exposing it to the action of pieces of skin, I found that it gave a much slighter colour to oxygenated sulphate of iron than an equal portion of a similar infusion which had becu immediately precipitated by solution of isinglass; but I am inclined to attribute this effect, not to any absorption of gallic acid by the skin, but rather to the decomposition of it by the long continued action of the atmosphere; for much insoluble matter had been preeipitated during the process of tanning, and the residuum contained a small portion of acetous acid.

In ascertaining the quantity of tannin in galls, I found that 500 grains of good Aleppo galls gave, by lixiviation with pure water till their soluble parts were taken up, and subsequent slow evaporation, 185 grains of solid matter. And this matter, examined by analysis, appeared to consist

Of tanuin

Of mucilage, and matter rendercd insoluble by evaporation - $\quad-\quad-\quad-12$

Of gallic acid, with a little cxtractive matter - 31

Remainder, calcareous earth and saline matter 12

The fluid obtained by the last lixiviation of galls, as 
M. Deycux observed, is pale green; and I am inclined to believe that it is chiefly a weak solution of gallate of lime. The ashes of galls, deprived of soluble matter, furnish a very considerable quantity of calcareous earth. And the property which M. Deyeux discovered in the liquor of the Jast lixiviations, of becoming red by the action of acids, and of regaining the green colour by means of alkalis, I have observed, more or less, in all the soluble compounds containing gallic acid and the alkaline earths.

[To be continued.]

IX. Extract from the third Volume of the Analyses of M. Klaproth.

[Continued from our last volume, p. 344.]

Anaiysis of the natural Muriate of Ammonia of Vesuvius.

A

A frer the eruption of Vesuvius in the year 1794, which continued several weeks, the vapours of the buming lava became in part condensed into concrete salts, which were found under different forms in the crevices and hollows of the upper scoriæ of the lava after it had cooled. The principal products of this natural sublimation are sal ammoniac and muriate of soda.

The sal ammoniac is sometimes pure, sometimes coloured yellow, and for the most part crystallised in prisms of four planes a little inclined, exceedingly brilliant and transparent.

Muriate of soda forms almost always shapeless strata of salt fibrous in their fracture: it is rarely pure, and for the most part mixed with an oxide of copper which commu. nicates to it a green colour more or less intense, and besprinkled in many places with small brilliant leaves of sparry iron.

The sublimated sal ammoniac ought, without doubt, to be considered as a product of the decomposition of water and atmospheric air in this grand chemical operation of nature.

It is not necessary to make a similar supposition to explain the formation of the muriate of soda. The sea water which penetrates to the focus of the volcano and concurs to its eruption contains it ready formed, while the decomposition of a part of this salt furnishes the free muriatic acid which forms muriate of ammonia.

M. Klaproth found in his analysis that this muriate is perfectly 